\title{
Electronic Transport Properties of Trigonal Graphene Nanoribbon with Different Edge Termination
}

\author{
LIU Wen ${ }^{* a}$, FANG Changfeng, ZHAO Jianhua, CHENG Jie and LIU Desheng ${ }^{\mathrm{b}}$ \\ Physics and Information Engineering Department, Jining University, Qufu, China \\ aliuwenjnxy@163.com \\ bliuds@sdu.edu.cn
}

\begin{abstract}
The electronic transport properties of trigonal graphene nanoribbon with different edge termination are studied with the first principle methods. It is found that edge termination plays an important role in electronic transport in these systems. The current of Fe-terminated nanoribbon increases more than two orders of magnitude compared with that of H-terminated systems. The reason of the current enhancement is attributed to the change of transmission spectra in the presence of different edge termination.
\end{abstract}

\section{Introduction}

Single layer graphene has attracted lots of attention due to its unique electrical and chemical properties. Graphene seems to be a promising material for building electronic devices which eventually could lead to 'carbon computers'. Owing to the advancement of experimental technology, it has become possible to introduce foreign atoms or tailor a graphene nanoribbon (GNR) into different shape[1,2]. Guo et al have investigated the electronic and magnetic properties of zigzag-GNR with protruded steps along their edges (ZS-GNRs) by extensive density functional theory calculations[3]. Their findings suggest that the ZS-GNRs are promising for designing versatile graphene-based devices. A spin-valve device based on dumbbell-shaped graphene nanoislands is studied by spin-unrestricted Hubbard model[4]. Inspired by strong mechanical stability of "Y"-shaped beams for building construction, Wang et al have designed a new class of quasi-1D graphene nanostructures, namely, tri-wing graphene (TWG) nanoribbons. By tailoring the wing structures, the TWGs can provide broader property tunability for nanoelectronic application[5]. An armchair graphene nanoribbon based electronic device with spin filtering and rectifying behavior is designed by means of molecular tailoring, and the electronic transport properties are calculated by a non-equilibrium Green function (NEGF) method combined with density functional theory (DFT). The results show that rectifying behavior can be observed in the junctions[6]. Rectifying behavior was further confirmed by BN-doping in trigonal graphene with zigzag edges[7]. By applying NEGF+DFT, Deng et al

*Corresponding author:liuwenjnxy@163.com 
have investigated the effects of two side groups, $\mathrm{NH} 2$ and $\mathrm{NO} 2$, on the electronic transport properties of the trigonal graphene flake. It has been found that the rectification rations and direction can be significantly tuned by the type and attached positions of side groups[8].

It has been reported that the electron and spin transport properties are strongly depending on the type of terminated atoms at the edge of GNR[9]. Motivated by that, we investigate the effect of Fe termination on the electronic properties of trigonal GNR with the first-principle method. The results indicate that Fe termination instead of $\mathrm{H}$ termination at the edge has significant effect on the electronic transport.

\section{Method and Model}

In this section, we report on the electronic transport property of H-terminated ZGNRs and Fe-terminated ZGNRs, which are denoted as Z-H and Z-Fe for short, respectively. We construct a two-probe molecular device which consists of three regions: the left electrode, the right electrode, and the center region. In presence of the applied bias $V_{b}$, the electrochemical potentials of the left and right electrode will be shifted up and down, respectively.

The geometry structures are first optimized with a residual force of $0.05 \mathrm{eV} / \AA$. After the structure relaxation, we calculate the electronic transport properties of the system by the well test ATK package ${ }^{[17]}$, which adopts NEGF method combined DFT. The double-zeta polarization (DZP) basis set for valence electrons is adapted. The Perdew- BurkeErnzerhof (PBE) formulation of the generalized gradient approximation (GGA) exchange correlation function is used. Core electrons are modeled with Troullier-Martins nonlocal pseudopotential. An energy cut off of $200 \mathrm{Ry}$ is used to define the real-space grid for numerical calculations in the electron density. The electronic Brillouin zone integration is sampled with a $1 \times 1 \times 100$ uniform k-point mesh. The current is calculated using the Landauer-Büttiker formula:

$$
I=\frac{2 e}{h} \int T\left(E, V_{b}\right)\left[f_{L}\left(E-\mu_{L}\right)-f_{R}\left(E-\mu_{R}\right)\right] d E
$$

where $T\left(E, V_{b}\right)$ is the transmission coefficient for electrons with energy $E$ at bias voltage $V_{b}, f_{\mathrm{L} / \mathrm{R}}$ the Fermi function, and $\mu_{\mathrm{L} / \mathrm{R}}$ the electrochemical potential of the left/right electrode.

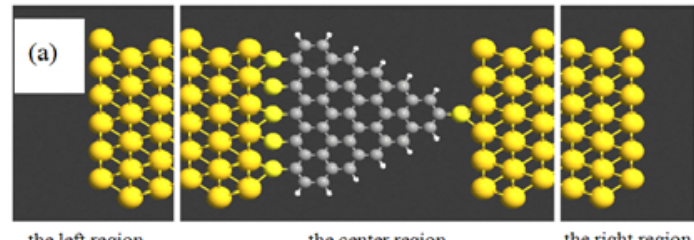

the left region

the center region

the right region

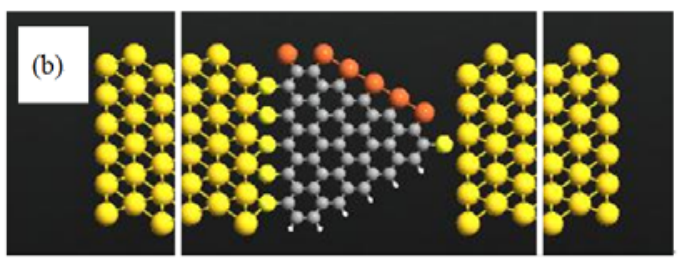

the left region

the center region

the right region

Fig. 1 The geometric structures (a) Z-H and (b) Z-Fe. 


\section{Results and Discussion}

Using first principles NEGF method, we show in Fig.2 the transmission coefficient $T$ at zero bias of $\mathrm{Z}-\mathrm{H}$. The distances between $\mathrm{S}$ atoms and the left and right electrodes are $2.0 \AA$ and $1.8 \AA$, respectively. From fig. 2 we can find that a broad transmission gap appears in the energy range $(-1.35 \mathrm{eV}, 1.3 \mathrm{eV})$, which indicates semiconductor transport behavior of the system. The peak around the Fermi level is so small that they nearly have no contribution to the electron transmission. Although two larger transmission peaks appear at $E=1.7 \mathrm{eV}$ and $1.9 \mathrm{eV}$. However, they are two far from the Fermi level and can not contribute to the transmission.

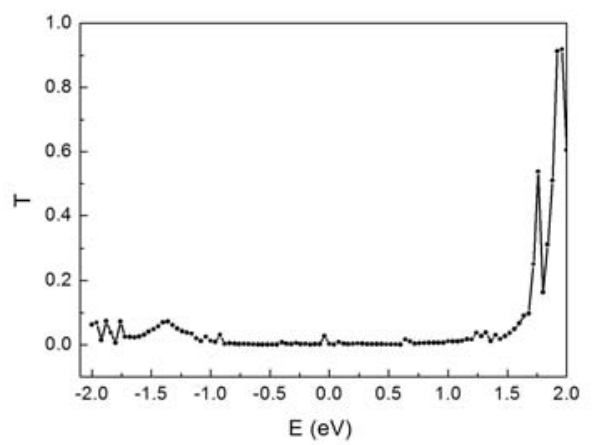

Fig.2 The transmission spectrum T(E) at zero bias for Z-H.

Fig. 3 shows the current as a function of the applied bias. As can be seen from the figure, once the bias is applied the current begins to increase slowly. Until the bias voltage increases up to about $1.3 \mathrm{eV}$, the enhancement of current takes on a trend of acceleration under the positive bias. In the case of negative bias, the situation is similar. As the energy goes beyond $1.4 \mathrm{~V}$, the negative differential resistance (NDR) phenomenon is observed. The current decreases when the bias increases from $V_{b}=1.4 \mathrm{~V}$ to $V_{b}=1.6 \mathrm{~V}$. Next we change the left and right molecule-electrode contact distances to $1.8 \AA \AA$ and $2.0 \AA \AA$, respectively, and find that NDR phenomenon still exists as the bias increases from $V_{b}=1.6 \mathrm{~V}$ to $V_{b}=1.8 \mathrm{~V}$. However, when we change contact distances to $1.9 \AA$ and $2.0 \AA$, the NDR phenomenon disappears in the energy range we studied. Thus one can expect that the NDR phenomenon in our trigonal graphene nanoribbon is related to the molecule-electrode contact distance. We change both

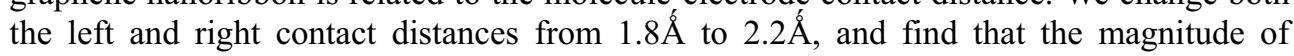
current does not differ obviously. The calculated I-V curves are depicted in fig.3(a). It can be found that in the distance range we studied, the I-V characteristic change slightly, i.e., the I-V characteristic is not sensitive to the distance we focus on. Due to the computation time restriction, a wider distance range remains for the forthcoming study. However, when the $\mathrm{H}$ atoms at one edge are substituted by $\mathrm{Fe}$ atoms the situation is totally different. In fig.3(b) we show the I-V curves for two Z-Fe system, in which the left and right contact distances are $1.8 \AA \AA$ and $2.0 \AA \hat{\text {, }}$, respectively. The current increases more than two orders of magnitude compared with the Z-H systems. The I-V curves of system Z-H are also depicted in fig.3(b) for comparison. 

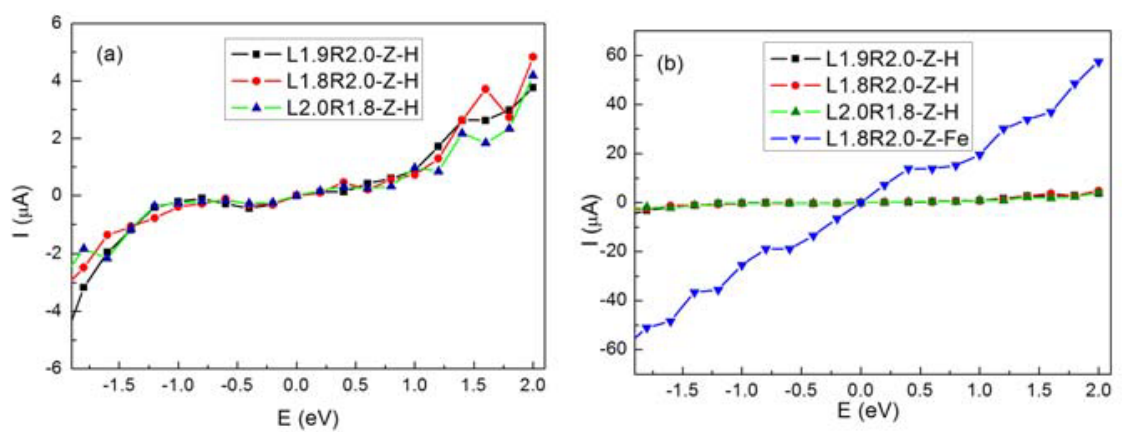

Fig.3 The I-V characteristic for system Z-H and Z-Fe with different contact distances.

To elucidate the effects of Fe-substitution on the electron transport, we give the transmission spectra $T\left(E, V_{b}\right)$ at different bias voltages in fig.4. As the current is obtained from the Landauer-Büttiker formula mentioned above, the magnitude of current in fact depends on the transmission coefficient in the integral regions, namely, the size of the integral area in the bias window. Therefore, to further understand the change of the current, we can turn to the bias dependence of $T\left(E, V_{b}\right)$ at different bias voltages as shown in fig.4. The dashed lines denote the corresponding bias windows at different biases. In the cases of $V_{b}=1.0 \mathrm{~V}$ for $\mathrm{Z}-\mathrm{H}$, a transmission peak enters the bias window and the total integral area of transmission coefficients enlarges. Therefore, the current increases compared with $V_{b}=0$. For Z-Fe, however, more peaks enter the bias window and the transmission coefficient is much larger than that of $\mathrm{Z}-\mathrm{H}$ at $V_{b}=1.0 \mathrm{~V}$, leading to the current increases by more than two orders of magnitude. When the bias is further increased to $2.0 \mathrm{~V}$, we can perform the same analysis.

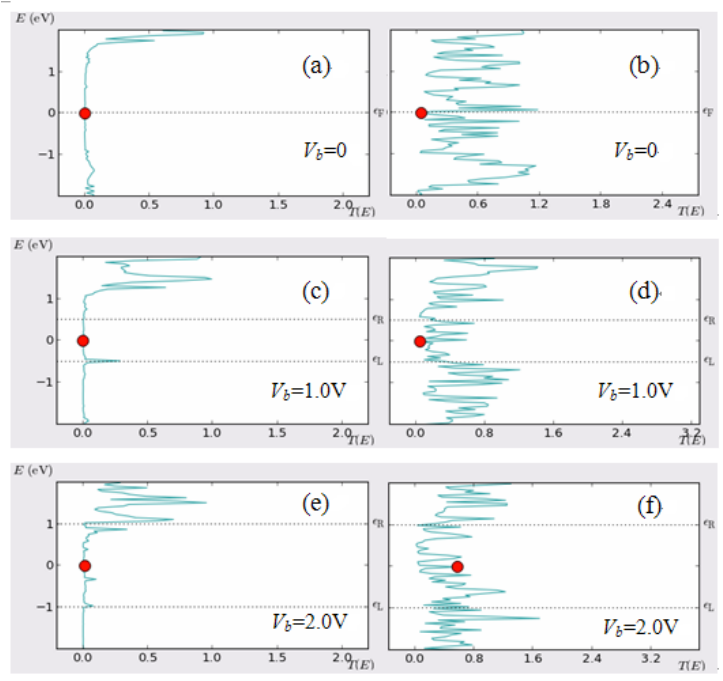

Fig. 4 Transmission spectra for Z-H (the left panel) and Z-Fe (the right panel) at different bias voltages $V_{b}=0,1.0 \mathrm{~V}$, and $2.0 \mathrm{~V}$.

\section{Summary}

We have investigated the electronic transport properties of H-terminated ZGNRs and 
Fe-terminated ZGNRs using NEGF method combined with DFT. The results indicate that edge termination has important influences on the electronic transport. The current of Fe-terminated nanoribbon is two orders of magnitude larger than that of H-terminated systems. The increase in current is ascribed to the enhancement of the transmission coefficient caused by Fe-termination instead of $\mathrm{H}$-termination.

\section{Acknowledgement}

This work was supported by the Natural Science Foundation of Shandong Province (Grant No. ZR2014AL013 and ZR2013AL006) and Shandong Province Higher Educational Science and Technology Program (Grant No. J13LJ53).

\section{References}

1. X. Li, X. Wang, L. Zhang, S. Lee and H. Dai, Ultrasmooth graphene nanoribbon semiconductors, Science. 319 (2008) 1229-1231.

2. H. T. Wang, Q. Wang, Y. Chang, et al., Doping monolayer graphene with single atom substitutions, Nano Lett. 12(2012)141-144.

3. W. Z. Wu, Z. H. Zhang, P. Lu, and W. L. Guo, Electronic and magnetic properties of zigzag graphene nanoribbons with periodic protruded edges, Phy. Rev. B, 82(2010)085425.

4. Z. L. Ma and W. D. Sheng, A spin-valve device based on dumbbell-shaped graphene nanoislands, Appl. Phys. Lett. 99(2011)083101

5. L. Y. Zhu, J. L. Wang, and T. T. Zhang, Mechanically robust tri-wing graphene nanoribbons with tunable electronic and magnetic properties. Nano Lett. 10(2010)494-498.

6. J. He and K. Q. Chen, Rectifying and perfect spin filtering behavior realized by tailoring graphene nanoribbons. J. Appl. Phys, 112(2012)114319.

7. X. Q. Deng, Z. H. Zhang, G. P. Tang, et al., Rectifying behaviors induced by BN-doping in trigonal graphene with zigzag edges, Appl. Phys. Lett. 100(2012)063107.

8. X. Q. Deng, G. P. Tang, and C. Guo, Tuning the electronic transport properties for a trigonal graphene flake, Phys. Lett. A. 376(2012)1839-1844.

9. C. Cao, L. N. Chen, and M. Q. Long, Electronic transport properties on transition-metal terminated zigzag graphene nanoribbons, Journal of Applied Physics. 111(2012)113708. 